\title{
Nucleotide Substitution Site
}

National Cancer Institute

\section{Source}

National Cancer Institute. Nucleotide Substitution Site. NCI Thesaurus. Code C157224.

The nucleotide position that is substituted in a nucleic acid variant. 